\title{
Is VVI pacing outmoded?
}

\author{
Anthony W Nathan, D Wyn Davies
}

Pacing is a common clinical procedure. The permanent implant rate in the United Kingdom is lower than in other Western countries. The last World Survey ${ }^{1}$ showed a new implant rate of 148 per million population per year (8346 systems) in the United Kingdom. This compares with 421 in Germany, 413 in France, 359 in the United States, and 175 in Spain. In the United Kingdom, the pacing mode was simple constant rate ventricular inhibited (VVI) ${ }^{2}$ in about $78 \%$, despite the widespread availability of more physiological systems. This proportion has not changed significantly in more recent years. It is our view that the simple VVI mode is not appropriate in 1992 and that almost every patient would be better served by an alternative mode, even though these other modes may take more time to implant and follow-up, may cost more, and require some expertise, which may easily be acquired.

\section{Indications for permanent pacing}

The major indications for pacing for bradycardia are symptom relief and the improvement of prognosis. Symptoms of bradycardias include syncope (Adams-Stokes' attacks), lesser degrees of disturbed consciousness, breathlessness, fatigue, and chest pain. Depending on the underlying pathology, other symptoms may be secondary to associated abnormal tachycardias or emboli. The prognosis is influenced by the underlying pathology. The major pathologies are sinoatrial disease $(31 \%$ of United Kingdom implants) and atrioventricular conduction abnormalities (54\%) as well as carotid sinus hypersensitivity. These entities may occur alone or in combination. Pacing may also be indicated for the termination of reentrant tachycardias but this is an uncommon indication and is beyond the scope of this article.

St Bartholomew's Hospital, London A W Nathan St Mary's Hospital, London D Wyn Davies

Correspondence to Dr Anthony W Nathan, Department of Cardiology, St Bartholomew's Hospital West Smithfield, London EC1A 7BE.

Accepted for publication 25 November 1991 endocardial pacing and sensing of the right atrium, right ventricle, or both are possible with programmable devices that can vary the pacing rate according to the patient's needs.
Simple ventricular demand pacemakers commit the patient to a constant pacing rate and are unresponsive to fluctuating metabolic needs. Atrioventricular synchrony is lost and retrograde atrioventricular conduction may occur producing unpleasant symptoms and deleterious haemodynamic effects (pacemaker syndrome). ${ }^{4}$ Symptoms of the pacemaker syndrome include breathlessness, weakness, dizziness, presyncope, pulsations in the neck and palpitation. Heldman et al randomised 40 patients blindly to VVI or dual chamber pacing in a crossover study. ${ }^{5}$ Pacemaker syndrome was recognised in $83 \%$ of patients in the VVI mode and was moderate or severe in $65 \%$ (intolerable in $42 \%$ ). Atrial pacing alone may be useful in some patients with sinoatrial disease.

To overcome problems associated with a constant pacing rate, more sophisticated pacemakers have been developed. One solution has been to control the pacing rate according to one of a multitude of physiological variables (rate responsive pacing). These include variables such as the QT interval, ${ }^{6}$ respiratory rate, ${ }^{7}$ minute ventilation, ${ }^{8}$ mixed venous temperature, ${ }^{9}$ or oxygen saturation, ${ }^{10}$ which reflect metabolic needs. Other sensors simply respond to varying levels of activity by detecting motion in different ways. ${ }^{112}$ All these systems have been shown to be clinically practical and useful, and implantation is usually identical to a standard single chamber system. There are problems with all of these systems, however; some of them may be specifically sensor related. ${ }^{13-15}$ Furthermore, patients with ventricular demand systems (VVIR) will also lose atrioventricular synchrony and may be subject to the pacemaker syndrome. In addition, some sensors are not ideal for atrial rate-responsive demand pacing (AAIR).

Another method of obtaining rate response is to sense the natural atrial rate. This requires a dual chamber system. Originally these systems could neither pace the atrium nor sense the ventricle (VAT mode). Then ventricular sensing was achieved (VDD mode), and now most dual chamber pacemakers are capable of operating in the "universal" DDD mode, which allows atrial synchronous ventricular pacing with additional demand atrial pacing and ventricular sensing. A more recent development has been the DDI mode which 
allows atrioventricular sequential pacing at a constant rate with both atrial and ventricular sensing but where ventricular pacing is not triggered by atrial sensing. The DVI mode, which has been superseded by DDI, was similar but without atrial sensing.

Conventional dual chamber pacemakers overcome many problems by providing rate response, maintaining atrioventricular synchrony, and preventing the pacemaker syndrome. Their value is limited when there is chronotropic incompetence or if there are persistent atrial arrhythmias. Problems specific to dual chamber pacemakers are related to implantation and to the increased complexity of the hardware. Two pacing leads are generally required, although single pass leads have occasionally been used. Refinements such as the development of thin bipolar leads with various fixation mechanisms have circumvented problems related to both implantation and atrial sensing so that most centres find little additional difficulty with dual chamber compared with single chamber implants. Joseph et $a l,{ }^{16}$ in a district general hospital, had a longterm success rate of $98 \%$ using modern atrial leads. Pacemaker mediated reentrant or "endless loop" tachycardia is a problem exclusive to dual chamber pacemakers. ${ }^{17}$ It occurs in some patients with retrograde conduction (from ventricle to atrium) through sensing of retrograde atrial activation, and is often at the upper rate limit of the pacemaker. However, modern pacemaker design has minimised this problem.

To cope with patients with chronotropic incompetence, some dual chamber pacemakers are now available with a non-atrial sensor thus offering, for example, DDDR or DDIR pacing. These pacemakers also allow selection of the ventricular rate responsive mode if, for example, atrial fibrillation develops in a patient already being paced for atrioventricular block.

\section{Choice of pacemaker}

SINOATRIAL DISEASE

Special considerations when pacing patients with sinoatrial disease include improving the overall prognosis, the prevention of atrial tachycardias, overcoming an increased risk of thromboembolic events, and the state of atrioventricular conduction (anterograde and retrograde). There are few data on overall mortality in this disorder, although Shaw et al described a survival rate similar to a matched control population. ${ }^{18}$ However, some of their patients had only mild sinoatrial abnormalities. Pacing did not seem to alter prognosis in this study, but most of the patients were paced only from the ventricle.

Rosenqvist $e t$ al showed that in patients free from atrial fibrillation at the time of implant, $47 \%$ of those paced from the ventricle (VVI) developed persistent atrial fibrillation compared with $6.7 \%(p<0.0005)$ paced with atrial inhibited pacemakers (AAI) over a four year follow up. ${ }^{19}$ The VVI group had a higher incidence of congestive heart failure $(37 \%)$ than the AAI group (15\%, p < 0.005), and a higher mortality (23\% compared with $8 \%$, $\mathrm{p}<0.05)$. Several other studies have also shown the value of pacing the atrium in preventing atrial fibrillation. ${ }^{20-22}$ The risk of systemic embolism probably influences the prognosis, and this is in turn linked to the development of supraventricular tachycardias, especially atrial fibrillation. Sutton and Kenny pooled data from several studies (853 patients) and showed a $1.6 \%$ incidence of systemic emboli in AAI paced patients compared with $13 \%$ in VVI paced patients $(p<0.001){ }^{23}$ Many patients with sinus node disease have intact retrograde atrioventricular conduction and are thus at risk from the pacemaker syndrome if paced from the ventricle alone. ${ }^{24}$ For all of these reasons, pacing systems for patients with sinus node disease should at least incorporate an atrial lead.

For single chamber pacing, the AAI mode may be used. If there is significant chronotropic incompetence, a rate responsive system (AAIR) can be chosen, ${ }^{25}$ although this imposes increased stress on atrioventricular conduction. Although many sensors are suitable for AAIR pacing, the QT interval is an obvious exception; atrial temperature based sensor systems can be unreliable, and even with activity based systems there have been problems.

AAI or AAIR pacing is inappropriate for patients with sinoatrial disease together with impaired atrioventricular conduction at the time of diagnosis. In addition, some of the patients with normal conduction at the time of implant may develop impairment of atrioventricular conduction with time. Rosenqvist et al documented the development of high grade atrioventricular block in 4 of 89 patients $(4.5 \%)$ paced from the atrium over a 4 year period. ${ }^{19}$ Rosenqvist and Obel surveyed published reports and found 28 studies, relating to 1878 patients followed for a median period of three years. ${ }^{26}$ The median prevalence of development of significant atrioventricular block was $2 \cdot 1 \%$, an annual incidence of $0.6 \%$. Thus there is a risk of developing significant atrioventricular block with time, but the risk is low.

The development of chronic atrial fibrillation will render atrial pacemakers ineffective, but many patients will have adequate ventricular response rates. Some suggest that dual chamber pacing should be the rule in patients with sinoatrial disorders because of these limitations, ${ }^{23}$ but this remains controversial.

If dual chamber pacing is chosen in patients with sinoatrial disease, care must be taken to select the correct mode. The high incidence of retrograde conduction in these patients means that there is often an ideal substrate for pacemaker mediated "endless loop" tachycardias. These may complicate DDD pacing so that DDI pacing is often preferred. The advent of dual chamber, sensor responsive pacemakers has allowed DDDR or DDIR pacing in these patients, with DDIR being ideal for many.

\section{ATRIOVENTRICULAR BLOCK}

The prognosis of patients with atrioventricular block is radically improved by even the simplest form of ventricular pacing. ${ }^{27}$ In certain 
cases this can be further improved by more sophisticated pacing, but in almost all cases the quality of life can be enhanced by the correct choice of pacemaker. ${ }^{28-30}$

The principal determinant of cardiac output in paced patients is the heart rate. ${ }^{31}$ Patients with rate responsive pacemakers perform better during exercise than those with simple VVI devices, whether or not the increase in rate is achieved synchronously (VAT, VDD, or DDD pacemakers) ${ }^{283032}$ or asynchronously (VVIR pacemakers).$^{33} 34$ Pacemakers that provide only atrioventricular synchrony without atrial tracking (DVI or DDI modes) provide only a small increase in exercise performance.

As comparable improvement is obtained with VVIR and dual chamber pacing, what governs the choice between them? Single chamber VVIR pacing is simple to implement and is the method of choice when chronic atrial arrhythmias are present, when an atrial lead would be ineffective. Dual chamber systems avoid the pacemaker syndrome, which is common in VVI or VVIR modes, and if sinus node function is normal, use the best possible sensor-atrial rate. However, "endless loop" tachycardias are a very occasional nuisance. Initial programming is also simpler with DDD systems, because VVIR pacemakers require the correct relation between the sensor and exercise performance to be ascertained. Overall, the prognosis of patients with atrioventricular block has not been shown to be significantly improved by chosing dual chamber rather than ventricular pacing. ${ }^{35}$ However, Alpert et al found improved one, three and five year survival in a subset of patients with congestive heart failure treated with DDD compared with VVI pacing. ${ }^{35}$ Kenny et al also found particular benefit could be gained from treating patients with angina with dual chamber devices rather than ventricular systems, provided the upper rate was limited to 110 beats per minute. ${ }^{36}$ Rate responsive dual chamber pacemakers offer complete flexibility for patients with atrioventricular block who have additional chronotropic incompetence.

\section{CAROTID SINUS SYNDROME}

In these patients, sinus bradycardia or arrest is common, as is atrioventricular block. These aspects can easily be treated with pacing. The vasodepressor element is present to a greater or lesser degree in most patients, and may be exacerbated by ventricular pacing. ${ }^{37}$ Intact retrograde conduction is common $(80 \%),{ }^{37}$ and therefore the pacemaker syndrome is likely with ventricular pacing. Hysteresis (a feature where the pacemaker has a faster pacing rate

Recommended pacing modes (codes are defined in the text)

\begin{tabular}{ll}
\hline Diagnosis & Optimal mode \\
\hline Sino-atrial disease & AAI/AAIR \\
Atrioventricular block & DDD/DDDR \\
Sinoatrial disease + persistent atrioventricular block & DDD/DDDR \\
Sinoatrial disease + occasional or threatened atrioventricular block & DDI/DDIR \\
Atrioventricular block + chronic atrial arrhythmias & VVIR \\
Carotid sinus syndrome & DDI \\
\hline
\end{tabular}

^If chronotropic incompetence is present. than sensing rate) may reduce but not abolish these problems. Dual chamber pacing is preferred for these patients. Because the atrial rate response to exercise is usually normal and retrograde conduction is common, the DDI mode is usually recommended.

\section{Intermittent conduction abnormalities}

Some patients may have been shown to have only occasional conduction abnormalities and sporadic symptoms. Likewise some patients without proven high grade conduction defects may be paced, either because of symptoms, which may be infrequent, or in an effort to improve prognosis. Pacemakers implanted in these patients should have hysteresis selected. It is tempting to use VVI pacemakers in such patients whatever the suspected conduction abnormality, but this can be a grave mistake. It is usual for abnormalities that present as occasional to become more frequent with the passage of time. Furthermore, those paced speculatively for, perhaps occasional syncope caused by suspected intermittent atrioventricular block, may turn out to have the carotid sinus syndrome and while a simple VVI pacemaker may prevent syncope in the former case it is unlikely to do so consistently in the latter.

\section{Patient assessment}

Each patient needs to be carefully evaluated before implantation to decide what is the optimal pacemaker. As well as routine clinical assessment a careful electrocardiographic diagnosis of the arrhythmias being treated is obviously necessary. Simple exercise testing may be necessary to determine whether or not the atria are capable of a satisfactory rate response. Ambulatory monitoring may be required in order to make a diagnosis, in order to examine rate response, and also to determine whether any tachycardias are present. Carotid sinus massage or tilt testing is occasionally useful, but electrophysiological testing is rarely necessary.

\section{Conclusions}

The correct pacing mode is usually determined by the site of the conduction abnormality and not by the underlying aetiology (table). Three simple rules should be adhered to when chosing the correct pacing mode:

(1) The atrium should be sensed and paced unless contraindicated (chronic atrial arrhythmias or rare anatomical considerations).

(2) The ventricle should be paced if atrioventricular block is present or is likely to develop.

(3) A rate responsive sensor should be added if there is chronotopic incompetence or if atrial activity is abnormal.

Ideally, no patient should receive a constant rate VVI pacemaker. General infirmity may encourage one or more of these rules to be broken, but if the rules are broken for purely 
budgetary reasons, this should be acknowledged as being the case. Only by doing this will optimal pacing facilities develop, and those in training may then understand clinical decisions to implant less than ideal pacemakers. Although a pacing service has to be provided within a limited budget, any saving made by choosing a cheaper pacing mode has to be set against limitations in the patient's quality and, in some cases, quantity of life. We, and many of our colleagues, do not always keep to our guidelines, but this is purely for fiscal reasonsit is important that we do not try to convince ourselves otherwise. When pacing practice is subjected to medical audit, the clinical outcome may be shown to outweigh any financial arguments.

1 World Survey Data. PACE 1987;10:769-77.

2 Bernstein AD, Camm AJ, Fletcher RD, et al. The NASPE BPEG generic pacemaker code for antibradyarrhythmia and adaptive-rate pacing and antitachyarrhythmia devices. PACE 1987;10:794-9.

3 Senning A. In discussion: Goott B, Miller FA. Prevention of posthypercapneic ventricular fibrillation in dogs. Thoracic Surg 1959;38:630-39.

4 Johnson AD, Laiken SL, Engler RL. Hemodynamic compromise associated with ventriculoatrial conduction following transvenous pacemaker placement. Am J Med lowing transvenc

5 Heldman D, Mulvihill D, Nguyen $\mathrm{H}$, et al. True incidence of pacemaker syndrome. PACE 1990;13:1742-50.

6 Donaldson RM, Fox K, Rickards AF. Initial experience with a physíological, rate responsive pacemaker. Br Med J 1983;286:667-71.

7 Rossi P, Aina F, Rognoni G, Occhetta E, Plicchi G, Prando $M D$. Increasing cardiac rate by tracking the respiratory rate. $P A C E$ 1984;7:1246-56.

8 Nappholtz T, Valenta H, Maloney J, Simmons T. Electrode configurations for a respiratory impedance measurement suitable for rate responsive pacing. $P A C E 1986 ; 9: 960-4$.

9 Alt E, Hirgstetter C, Heinz M, Blomer H. Rate control of physiologic pacemakers by central venous blood temperature. Circulation 1986;73:1206-12.

10 Foerestrand S, Skadberg BT, Anderson K, Ohm OJ. Acute clinical testing and follow-up of a rate-variable pacemaker clinical testing and follow-up of a rate-variable pacemaker controlled by central venous oxyg

11 Humen DP, Kostuk WJ, Klein GJ. Activity-sensing, rateresponsive pacing: improvement in myocardial performance with exercise. PACE 1985;8:52-9.

12 Lau C-P, Tse W-S, Camm AJ. Clinical experience with Sensolog 703: a new activity sensing rate responsive pacemaker. $P A C E$ 1988;11:1444-55.

13 Lau C-P, Mehta D, Toff WD, Stott RJ, Ward DE, Camm AJ. Limitations of rate responsive of an activity-sensing rate-responsive pacemaker to different forms of activity. PACE 1988;11:141-50.

14 Rossi P, Prando MD, Magnani A, Aina F, Rognoni G, Occhetta E. Physiological sensitivity of respiratorydependent cardiac pacing: four-year follow-up. $P A C E$ dependent cardiac

15 Lau CP, Antoniou A, Ward DE, Camm AJ. Reliability of minute ventilation as a parameter for rate responsive pacing. $P A C E$ 1989;12:321-30.

16 Joseph S, Crowther A, Bahri A, Rose A. Positive fixation electrodes 6-12 years on, a $98 \%$ success story [abstract] PACE 1991;14:640

17 Rubin JW, Frank MJ, Boineau JP, Ellison RG. Current physiologic pacemakers: a serious problem with a new device. Am J Cardiol 1983;52:88-91.

18 Shaw DB, Holman RR, Gowers JI. Survival in sinoatrial disorder (sick-sinus syndrome). Br Med J 1980;I:139-41.
19 Rosenqvist M, Brandt J, Schuller H. Long-term pacing in sinus node disease: effects of stimulation mode on car-
diovascular morbidity and mortality. Am Heart J diovascular mor

20 Markewitz A, Schad N, Hemmer W, Bernheim C, Ciavolella $\mathrm{M}$, Weinhold $\mathrm{C}$. What is the most appropriate stimulation mode in patients with sinus node dysfunction? $P A C E$ 1986;9:1115-20.

21 Attuel P, Pellerin D, Mugica J, Coumel P. DDD pacing: an effective treatment modality for recurrent atrial arrhythmias. PACE 1988;11:1647-54.

22 Shandling AH, Feuer JM, Atwood JE, et al. The effect of cardiac pacing mode on the long-term development of atrial fibrillation [abstract]. J Am Coll Cardiol 1989;13:217A.

23 Sutton $R$, Kenney R-A. The natural history of sick sinus syndrome. PACE 1986;9:1110-4.

24 Furman S, Hayes DL, Holmes DR. A Practice of Cardiac Pacing. Mount Kisco: Futura, 1986:60

25 Rosenqvist M, Ahren C, Norlander R, Ryden L, Schuller H. Atrial rate-responsive pacing - effect on exercise capacity [abstract]. PACE 1988;11:514

26 Rosenqvist M, Obel IWP. Atrial Pacing and the risk for AV block: is there a time for change in attitude? $P A C E$ 1989;12:97-101.

27 Siddons $\mathrm{H}$. Deaths in long-term paced patients. Br Heart J 1974;36:1201-9.

28 Sutton R, Perrins EJ, Morley C, Chan SL. Sustained improvement in exercise tolerance following physiological cardiac pacing. Eur Heart J 1983;4:781-5.

29 Boon NA, Frew AJ, Johnston JA, Cobbe SM. A comparison of symptoms and intra-arterial ambulatory blood pressure during long term dual chamber atrioventricular synchronous (DDD) and ventricular demand (VVI) pacing. $\mathrm{Br}$ ous (DDD) and vent

30 Fromer M, Kappenberger L, Babotai I. Subjective and objective response to single versus dual chamber pacing. $J$ Electrophysiol 1987;1:343-9.

31 Goldreyer BN. Physiologic pacing: the role of AV synchrony. $P A C E$ 1982;5:613-5.

32 Fananpazir L, Bennett DH, Monks P. Atrial synchronized ventricular pacing: contribution of the chronotropic response to improved exercise performance. PACE 1983;6:601-8.

33 Smedgard P, Kristensson BE, Kruse I, Ryden L. Rateresponsive pacing by means of activity sensing versus single rate ventricular pacing: a double-blind cross-over study. $P A C E$ 1987;10:902-15.

34 Lipkin DP, Buller N, Frenneaux $M$, et al. Randomised crossover trial of rate responsive Activitrax and conven1987;58:613-6.

35 Alpert MA, Curtis JJ, Sanfelippo JF, et al. Comparative survival after permanent ventricular and dual chamber pacing for patients with chronic high degree atrioventricular block with and without preexistent congestive heart failure. J Am Coll Cardiol 1986;7:925-32.

36 Kenny RA, Ingram A, Mitsuoka T, Walsh K, Sutton R Optimum pacing mode for patients with angina pectoris. Br Heart J 1986;56:463-8.

37 Morley CA, Perrins EJ, Grant P, Chan SL, McBrien DJ, Sutton R. Carotid sinus syncope by pacing: analysis of persistent symptoms and role of atrioventricular sequential pacing. Br Heart J 1982;42:411-8.

\section{Addendum}

Since the submission of this paper, a working party of the British Pacing and Electrophysiology Group have published Recommendations for pacemaker prescription for symptomatic bradycardia $(B r$ Heart $J$ $1991 ; 66: 185-91)$. We agree with their recommendations and think that our point of view is fully compatible with their's. 